\title{
A DISCRETE APPROACH TO MONOTONICITY OF ZEROS OF ORTHOGONAL POLYNOMIALS
}

\author{
MOURAD E. H. ISMAIL AND MARTIN E. MULDOON \\ To our friend P. G. Rooney on the occasion of his 65 th birthday
}

\begin{abstract}
We study the monotonicity with respect to a parameter of zeros of orthogonal polynomials. Our method uses the tridiagonal (Jacobi) matrices arising from the three-term recurrence relation for the polynomials. We obtain new results on monotonicity of zeros of associated Laguerre, Al-Salam-Carlitz, Meixner and Pollaczek polynomials. We also derive inequalities for the zeros of the Al-Salam-Carlitz and Meixner polynomials.
\end{abstract}

\section{INTRODUCTION}

The recurrence relation

$$
x s_{n}(x)=\beta_{n} s_{n+1}(x)+\gamma_{n} s_{n}(x)+\delta_{n} s_{n-1}(x), \quad n=0,1, \ldots,
$$

for a system of orthogonal polynomials gives rise to the tridiagonal (Jacobi) matrix

$$
H=\left[h_{i j}\right]=\left[\begin{array}{ccccc}
\gamma_{0} & \beta_{0} & 0 & 0 & \cdots \\
\delta_{1} & \gamma_{1} & \beta_{1} & 0 & \cdots \\
0 & \delta_{2} & \gamma_{2} & \beta_{2} & \cdots \\
\cdots & \cdots & \cdots & \cdots & \ldots \\
\cdots & \ldots & \ldots & \ldots & \cdots
\end{array}\right] .
$$

The purpose of this work is to present a discrete approach to the question of monotonicity of zeros of a parameter-dependent family of orthogonal polynomials as functions of the parameter involved. One may think of the parameter dependence as a perturbation. An outline of our approach will be given in $\S 2$. When the coefficients $\beta_{n}, \gamma_{n}, \delta_{n}$ in (1) depend on a parameter $\tau$, the zeros of the $s_{n}(x)$ 's will depend also on $\tau$ and we are particularly interested in the behavior of the zeros as functions of $\tau$. The Hellmann-Feynman theorem, Theorem 2.1, gives a formula for the derivative of a zero of $s_{N}(x)$ with respect to $\tau$. The definiteness of the derivative of the $N \times N$ truncation of $H$ implies the

Received by the editors September 12, 1989.

1980 Mathematics Subject Classification. Primary 33A65; Secondary 15A42, 39A12.

Key words and phrases. Orthogonal polynomials, zeros, monotonicity, recurrence relations, Jacobi matrices.

The authors' work was supported by grants from the National Science Foundation and from the Natural Sciences and Engineering Research Council (Canada). 
monotonicity of the zeros of $s_{N}(x)$. The question of definiteness turns out to be equivalent to the question of whether certain sequences are chain sequences in the terminology of Chihara [6]. We discuss this connection and provide alternative criteria for monotonicity of a zero in $\S 3$.

In the remaining sections, we illustrate the method of $\S 2$ by several examples: the associated Laguerre polynomials, the Al-Salam-Carlitz polynomials, the Meixner polynomials, and the Pollaczek polynomials. We also derive inequalities for zeros of Meixner and Pollaczek polynomials. As a by-product we determine the rate of convergence of zeros of Meixner polynomials $m_{n}(x ; \beta, c)$ to the corresponding zeros of Laguerre polynomials $L_{n}^{(\beta-1)}(x)$ as $c \rightarrow 1^{-}$.

It is worth noting that other approaches to monotonicity of zeros of orthogonal polynomials use very special properties of the polynomials involved. For example, Markov's theorem [21, Theorem 6.12.1; 9, p. 133, problem 15] uses the knowledge of the weight function. Sturmian arguments $[21,18,19]$ use second-order differential equations. General orthogonal polynomials do not satisfy Sturmian second-order differential equations. Weight functions are not easy to compute (see the discussion in [14]) and even if the weight function is known, it may not satisfy the hypotheses of Markov's theorem, especially if, as in the examples in [2], the measure of orthogonality has a discrete part where the location of the masses depends on the parameter.

\section{Foundations}

There are two basic ingredients to our approach. The first is the finitedimensional version of the Hellmann-Feynman theorem concerning the derivative of an eigenvalue with respect to a parameter. The second is the relation between the interval of orthogonality of a system of orthogonal polynomials and the positive definiteness of certain matrices related to the tridiagonal matrix of the system.

The following formulation of the Hellmann-Feynman theorem will be sufficient for our purposes. For references to stronger versions, see [17].

Theorem 2.1 (see, e.g, [17]). Let $H(\tau)$ be an $n \times n$ Hermitian matrix whose entries have continuous first derivatives with respect to a parameter $\tau$ for $\tau \in$ $T:=\left(\tau_{1}, \tau_{2}\right)$. Let $\lambda_{1}(\tau)<\lambda_{2}(\tau)<\cdots<\lambda_{n}(\tau)$ be the eigenvalues of $H(\tau)$ and let $\mathscr{U}_{1}, \ldots, \mathscr{U}_{n}$ be the corresponding eigenvectors. If $\partial H(\tau) / \partial(\tau)$ is the matrix formed by the derivatives of the entries of $H(\tau)$, then

$$
\frac{\partial \lambda_{j}}{\partial \tau}=\frac{\mathscr{U}_{j}^{T}(\partial H / \partial \tau) \mathscr{U}_{j}}{\mathscr{U}_{j}^{T} \mathscr{U}_{j}}, \quad j=1, \ldots, n .
$$

An immediate consequence is the following:

Corollary 2.2. Let the assumptions of Theorem 2.1 hold. If $\partial H / \partial \tau$ is positive (negative) definite, then the jth eigenvalue of $H(\tau)$ is a strictly increasing (decreasing) function of $\tau, j=1, \ldots, n$. 
Note that we can replace "positive" ("negative") in Corollary 2.2 by "semipositive" ("seminegative") and conclude that the eigenvalues are nondecreasing (nonincreasing) functions of $\tau$. Corollary 2.2 is Theorem V.2.3 in Atkinson's book [4]. An equivalent assertion is that if $A$ and $B$ are Hermitian matrices and $B-A$ is positive definite then each eigenvalue of $B$ is strictly larger than the corresponding eigenvalue of $A$. Proofs of the latter assertion are in [11, Corollary $7.7 .4 ; 20$, p. 475].

Let $\left\{s_{n}(x)\right\}$ be a family of polynomials such that $s_{n}(x)$ is of precise degree $n, n=0,1, \ldots$, and

$$
s_{0}(x)=1, \quad s_{1}(x)=\left(x-\gamma_{0}\right) / \beta_{0} .
$$

Recall that such a family of polynomials is orthogonal with respect to a positive measure with infinite support and finite moments if and only if it satisfies a three-term recurrence relation of the form

$$
x s_{n}(x)=\beta_{n} s_{n+1}(x)+\gamma_{n} s_{n}(x)+\delta_{n} s_{n-1}(x), \quad n=1,2, \ldots,
$$

with

$$
\beta_{n} \delta_{n+1}>0, \quad n=0,1, \ldots
$$

The polynomials $\left\{s_{n}(x)\right\}$ are orthogonal with respect to a positive measure $\mu$ which we shall normalize by

$$
\int_{\mathscr{R}} d \mu(t)=1 .
$$

We associate with (2) and (3) the infinite tridiagonal matrix

$$
H=\left[h_{i j}\right]=\left[\begin{array}{ccccc}
\gamma_{0} & \beta_{0} & 0 & 0 & \cdots \\
\delta_{1} & \gamma_{1} & \beta_{1} & 0 & \cdots \\
0 & \delta_{2} & \gamma_{2} & \beta_{2} & \cdots \\
\cdots & \cdots & \cdots & \cdots & \cdots
\end{array}\right]
$$

and we use the notation $H_{N}$ for the $N \times N$ truncation of $H$. We have

$$
H_{N}\left[\begin{array}{c}
s_{0} \\
s_{1} \\
\cdots \\
\cdots \\
\cdots \\
s_{N-1}
\end{array}\right]=x\left[\begin{array}{c}
s_{0} \\
s_{1} \\
\cdots \\
\cdots \\
\cdots \\
s_{N-1}
\end{array}\right]
$$

if and only if $x$ is a zero of $s_{N}(x)$. This identifies the zeros of the polynomials $s_{N}(x)$, which are real and simple, as eigenvalues of the matrix $H_{N}$.

In what follows we will suppose that $\left\{s_{n}(x)\right\}$ satisfies (2) and (3) and assume that $\beta_{n}, \gamma_{n}$, and $\delta_{n}$ are differentiable functions of a parameter $\tau, 0 \leq n \leq N$, and we will be interested in using Theorem 2.1 to get a formula for the derivative of a zero of $s_{N}(x)$ with respect to $\tau$ and hence to discover conditions under which the zeros are monotonic functions of $\tau$. 
This problem has been considered by the first author [12] who used a slight generalization of Theorem 2.1 with nonsymmetric $H(\tau)$. This gives useful information in some cases, by showing that the entries in $\mathscr{U}_{j}^{T}(\partial H / \partial \tau) \mathscr{U}_{j}$ are all positive. Thus, for example, in the case of birth and death process polynomials $\left(\gamma_{n}=-\beta_{n}-\delta_{n}\right)$ one can get information on the monotonicity of the largest and smallest zeros in this way [12, Theorems 1, 2, and 3]. In the case of a class of polynomials considered by Dickinson, Pollack, and Wannier, $\beta_{n}=\delta_{n}$ and $\gamma_{n}=0$, one may get information on all the zeros in this way [12, Theorem 4].

Here we do not wish to make, at the outset, any special assumptions about the coefficients in the recurrence relation. However we will symmetrize the situation by considering, instead of $\left\{s_{n}(x)\right\}$, the corresponding orthonormal polynomials which are given by

$$
p_{0}(x)=1, \quad p_{n}(x)=\left(\frac{\beta_{0} \beta_{1} \cdots \beta_{n-1}}{\delta_{1} \delta_{2} \cdots \delta_{n}}\right)^{1 / 2} s_{n}(x),
$$

and which satisfy

$$
\begin{gathered}
p_{0}(x)=1, \quad x p_{n}(x)=a_{n} p_{n+1}(x)+b_{n} p_{n}(x)+a_{n-1} p_{n-1}(x), \\
n \geq 0, \quad a_{-1} p_{-1}(x):=0,
\end{gathered}
$$

where

$$
a_{n}=\sqrt{\beta_{n} \delta_{n+1}}, \quad b_{n}=\gamma_{n}, \quad n \geq 0 .
$$

This identifies the zeros of the polynomial $p_{N}(x)$ (or of $s_{N}(x)$ ) as eigenvalues of the Hermitian matrix $A_{N}$, the $N \times N$ truncation of the matrix

$$
A=\left[a_{i j}\right]=\left[\begin{array}{ccccc}
b_{0} & a_{0} & 0 & 0 & \cdots \\
a_{0} & b_{1} & a_{1} & 0 & \cdots \\
0 & a_{1} & b_{2} & a_{2} & \cdots \\
\cdots & \cdots & \cdots & \cdots & \cdots
\end{array}\right] .
$$

Theorem 2.1 may be applied directly in this setting and will give monotonicity of the zeros as functions of $\tau$, provided that $\partial A_{N} / \partial \tau$ is a definite matrix. We summarize our findings so far in the following theorem.

Theorem 2.3. Let $\left\{s_{n}(x)\right\}$ satisfy (2) and (3) and assume that, for $n=0,1, \ldots$, $N-1, \beta_{n}, \gamma_{n}$, and $\delta_{n}$ are differentiable functions of a parameter $\tau, \tau \in$ $\left(\tau_{1}, \tau_{2}\right):=T$. If $(4)$ holds for $n=0,1, \ldots, N-1$ and if the matrix

$$
\left[\frac{\partial}{\partial \tau} \sqrt{\beta_{i} \delta_{i+1}} \delta_{i, j-1}+\frac{\partial}{\partial \tau} \gamma_{i} \delta_{i j}+\frac{\partial}{\partial \tau} \sqrt{\beta_{i-1} \delta_{i}} \delta_{i, j+1}\right], \quad i, j=0, \ldots, N-1,
$$

is positive (negative) definite, then the zeros of $s_{0}(x), \ldots, s_{N}(x)$ are strictly increasing (decreasing) functions of $\tau, \tau \in T$.

In the application of Theorem 2.3, it is useful to have some methods of determining whether a symmetric tridiagonal matrix is positive definite. It is clear that if a symmetric tridiagonal matrix $A$ is positive definite, then any symmetric tridiagonal matrix obtained by increasing some of the diagonal entries 
and decreasing (in absolute value) some of the off-diagonal entries of $A$ is also positive definite. This is because

$$
\operatorname{det} A_{N}=b_{N-1} \operatorname{det} A_{N-1}-a_{N-2}^{2} \operatorname{det} A_{N-2} \text {. }
$$

We state this formally as follows.

Theorem 2.4. Let $A$ and $B$ be symmetric tridiagonal $N \times N$ matrices. Let $A$ be positive definite and let

$$
\frac{b_{i, i+1}^{2}}{b_{i i} b_{i+1, i+1}} \leq \frac{a_{i, i+1}^{2}}{a_{i i} a_{i+1, i+1}}, \quad i=0 \ldots, N-2 .
$$

Then $B$ is also positive definite.

In order to have a supply of positive definite matrices, to use as comparison matrices in the foregoing theorem, it is first worth noting that the matrix

$$
A_{N}=\left[\begin{array}{ccccc}
1 & 1 / 2 & 0 & \ldots & 0 \\
1 / 2 & 1 & 1 / 2 & \ldots & 0 \\
0 & 1 / 2 & 1 & \ldots & 0 \\
\vdots & \vdots & \vdots & \ddots & \vdots \\
0 & 0 & 0 & \ldots & 1
\end{array}\right]
$$

is positive definite. This follows from $\operatorname{det} A_{N}=(N+1) / 2^{N}$ which can be proved by induction on $N$ on noting that $\operatorname{det} A_{1}=1$, $\operatorname{det} A_{2}=3 / 4$, and, on using (6), $\operatorname{det} A_{N}=\operatorname{det} A_{N-1}-\frac{1}{4} \operatorname{det} A_{N-2}$. Another source of positive definite tridiagonal matrices is the tridiagonal matrices associated with polynomial systems whose intervals of orthogonality are contained in $[0, \infty)$. To explain this we need the concept of true interval of orthogonality.

Definition 2.5. Let $s_{n}(x)$ be an infinite sequence of orthogonal polynomials and let $x_{n, 1}<x_{n, 2}<\cdots<x_{n, n}$ be the zeros of $s_{n}(x), n>0$. The true interval of orthogonality is defined as $[\xi, \eta]$ where

$$
\xi=\lim _{n \rightarrow \infty} x_{n, 1}, \quad \eta=\lim _{n \rightarrow \infty} x_{n, n} .
$$

If $\left\{s_{n}(x) \mid 0 \leq n \leq N\right\}$ is a finite family of orthogonal polynomials, then we take

$$
\xi=x_{N, 1}, \quad \eta=x_{N, N} \cdot
$$

Both $\xi$ and $\eta$ exist in the extended real number system since $\left\{x_{n, 1}\right\}$ is a strictly decreasing, and $\left\{x_{n, n}\right\}$ a strictly increasing, sequence.

Theorem 2.6. Let $\left\{s_{n}(x)\right\}$ satisfy (2) and (3) and let the positivity condition (4) hold. Then an interval $[a, b]$ contains the interval of orthogonality $[\xi, \eta]$ of $\left\{s_{n}(x)\right\}$ if and only if $A_{N}-a I$ and $b I-A_{N}$ are positive definite for each $N \geq 1$. Proof. We have $\xi \geq a$ if and only if the eigenvalues of $A_{N}$ are $\geq a$, that is if and only if the eigenvalues of $A_{N}-a I$ are $\geq 0$, which is true if and only if 
$A_{N}-a I$ is positive definite. Similarly, we see that $\eta \leq b$ is equivalent to the positive definiteness of $b I-A_{N}$ for every $N$.

Theorem 2.6 is well known and is useful in providing a supply of positive definite tridiagonal matrices to be used in Theorem 2.4. If we know that the true interval of orthogonality of the system $\left\{s_{n}(x)\right\}$ is contained in $[a, b]$ we will have positive definite matrices $A_{N}-a I$ and $b I-A_{N}$. We may be able to use one of these, possibly with a different value of $\tau$ with which to compare $\partial A / \partial \tau$. We will use this technique to prove Theorem 5.1 on the monotonicity of the zeros of the Al-Salam-Carlitz polynomials.

\section{THE CONNECTION WITH CHAIN SEQUENCES}

The question of the positive definiteness of infinite tridiagonal matrices is intimately connected with the concept of chain sequences in the terminology of, e.g., [6].

Definition 3.1. A finite or infinite sequence $\left\{a_{n} \mid n>0\right\}$ is called a chain sequence if there exists another sequence $\left\{g_{n} \mid n \geq 0\right\}$ such that $a_{n}=$ $g_{n}\left(1-g_{n-1}\right), n>0$, with $0 \leq g_{0}<1$ and $0<g_{n}<1$ for $n>0$. The sequence $\left\{g_{n}\right\}$ is called a parameter sequence for the sequence $\left\{a_{n}\right\}$.

The following theorem is due to Wall and Wetzel [23]; see also [22].

Theorem 3.2. Let $B=\left[b_{i, j}\right]$ be an infinite real tridiagonal matrix

$$
b_{i, j}=c_{i-1} \delta_{i, j+1}+d_{i} \delta_{i, j}+c_{i} \delta_{i, j-1}, \quad i, j \geq 0,
$$

and assume $c_{i} \neq 0, \quad i=0, \ldots, N-2$. Let $B_{N}$ be the $N \times N$ truncation of $B$. Then $B_{N}$ is positive definite if and only if $d_{i}>0, i=0,1, \ldots, N-1$, and $\left\{c_{0}^{2} / d_{1} d_{0}, \ldots, c_{N-2}^{2} / d_{N-1} d_{N-2}\right\}$ is a chain sequence.

Thus the question of whether a symmetric tridiagonal matrix is positive definite reduces to the question of whether a certain related sequence is a chain sequence. The fact that the constant sequence $\{1 / 4\}$ is a chain sequence is equivalent to the positive definiteness of the matrix in equation (7). It may be useful also to record that the sequence whose $n$th element is $1 / 4+1 /[16 n(n+1)]$ is a chain sequence; see [6, p. 98].

We observe that Theorem 2.4 asserts that a sequence of positive numbers is itself a chain sequence if it is dominated by a chain sequence [6, Theorem 5.7, p. 97].

Our Theorem 2.3 may be rephrased as a theorem involving chain sequences in the following way:

Theorem 3.3. Let $\left\{s_{n}(x)\right\}$ satisfy (2) and (3) and assume that, for $n=0,1, \ldots$, $N-1, \beta_{n}, \gamma_{n}$, and $\delta_{n}$ are differentiable functions of a parameter $\tau, \tau \in$ $\left(\tau_{1}, \tau_{2}\right):=T$. If (4) holds for $n=0,1, \ldots, N-1$ and if $\alpha_{1}, \alpha_{2}, \ldots, \alpha_{N-1}$ is a finite chain sequence, where

$$
\alpha_{i}:=\left[\frac{\partial}{\partial \tau} \sqrt{\beta_{i} \delta_{i+1}}\right]^{2} /\left[\frac{\partial \gamma_{1}}{\partial \tau} \frac{\partial \gamma_{i-1}}{\partial \tau}\right], \quad i=0, \ldots, N-2,
$$


and if $\partial \gamma_{i} / \partial \tau>0(<0), i=0,1, \ldots, N-1$, then the zeros of $s_{0}(x)$, $\ldots, s_{N}(x)$ are strictly increasing (decreasing) functions of $\tau, \tau \in T$.

Chihara [5] pointed out the connection between chain sequences and the true interval of orthogonality of a family of orthogonal polynomials. His result follows from Theorems 2.6 and 3.2 but his proof is different. Although he stated his results for infinite families of orthogonal polynomials, his proofs are applicable to the case of finite families. The reader may note that the definitions in [5 and 6, p. 29] of the true interval of orthogonality of a family of polynomials require that family to be infinite, while our Definition 2.5 allows for finite families.

\section{Application to LaguerRe polynomials AND ASSOCIATED LAGUERRE POLYNOMIALS}

The associated Laguerre polynomials $\left\{L_{n}^{\alpha}(x ; c)\right\}[10,3]$ are generated by

$$
\begin{gathered}
L_{0}^{\alpha}(x ; c)=1, \quad L_{1}^{\alpha}(x ; c)=(\alpha+1-x+2 c) /(c+1), \\
x L_{n}^{\alpha}(x ; c)=-(n+c+1) L_{n+1}^{\alpha}(x ; c)+(2 n+2 c+\alpha+1) L_{n}^{\alpha}(x ; c) \\
-(n+c+\alpha) L_{n-1}^{\alpha}(x ; c), \quad n=1,2, \ldots
\end{gathered}
$$

We wish to investigate the dependence of the zeros of $\left\{L_{n}^{\alpha}(x ; c)\right\}$ on the parameter $\alpha$. The associated Laguerre polynomials provide an example of birth and death process polynomials, since $\gamma_{n}=-\beta_{n}-\delta_{n}$. Theorem 1 of [12] shows that the largest zero of such a polynomial increases with $\alpha, \alpha>0$. We will see that this increasing property holds for all the zeros.

In the notation of $\S 2$ we have $\beta_{n}=-n-c-1, \gamma_{n}=2 n+2 c+\alpha+1, \delta_{n}=$ $-n-c-\alpha$, the entries of the matrix $A_{N}$ are of the form

$$
\begin{aligned}
a_{i, j}= & \sqrt{(i+c+1)(i+c+\alpha+1)} \delta_{i, j-1} \\
& +(2 i+2 c+\alpha+1) \delta_{i, j}+\sqrt{(i+c)(i+c+\alpha)} \delta_{i, j+1},
\end{aligned}
$$

and so the entries of the matrix $\partial A_{N} / \partial \alpha$ are of the form

$$
c_{i, j}=\frac{\sqrt{i+c+1}}{2 \sqrt{i+c+\alpha+1}} \delta_{i, j-1}+\delta_{i, j}+\frac{\sqrt{i+c}}{2 \sqrt{(i+c+\alpha)}} \delta_{i, j+1} .
$$

Now, for $\alpha \geq 0$ and $c>-1$, this matrix is clearly positive definite, by comparison with the matrix in (7), using Theorem 2.4. This shows that for $\alpha \geq 0, \quad c>-1$, the zeros of the associated Laguerre polynomial increase with the parameter $\alpha$.

In the special case $c=0$, we have the classical Laguerre polynomials $\left\{L_{n}^{\alpha}(x)\right\}$ and the result just obtained shows that their zeros increase with $\alpha, \alpha \geq 0$. Actually the stronger result that the zeros increase with $\alpha, \alpha>-1$, can be obtained by Markoff's theorem [21, Theorem 6.12.1], by the method of Stieltjes $[21, \S 6.22]$ or, most simply, by the elegant electrostatic interpretation of the zeros [21, Theorem 6.7.3]. We point out, however, that these methods are 
based on knowing the weight function or the differential equation satisfied by the polynomials and are not obviously applicable in the case $c \neq 0$. We remark that the weight function for the associated Laguerre polynomials is (see [3]) $x^{\alpha} e^{-x}\left|\psi\left(c, 1-\alpha, x e^{-i \pi}\right)\right|^{-2}, \psi$ being the Tricomi $\psi$ function [7] and we know of no way to express the derivative with respect to a parameter of the Tricomi $\psi$ function in terms of simple special functions. Furthermore, if $c>$ $-1, \alpha+c>-1$ but $1+\alpha+2 c<0$, the measure of orthogonality of the associated Laguerre polynomials has a discrete mass which depends on $\alpha$, and Markov's theorem is not applicable. The associated Laguerre polynomials do not satisfy a second-order differential equation of Sturm-Liouville type. They satisfy a fourth-order differential equation with polynomial coefficients [10] which does not seem amenable to a Sturmian approach.

In the case of the usual Laguerre polynomials $(c=0)$, Theorem 2.1 may be used to provide a compact integral representation, (8) below, for the derivative of a zero $\xi$ of $L_{n}^{\alpha}(x)$ with respect to $\alpha$. In this case

$$
\mathscr{U}^{T}=\left(u_{0}, \ldots, u_{n-1}\right) \text {, }
$$

where

$$
u_{k}=(-1)^{k} \sqrt{k ! /(\alpha+1)_{k}} L_{k}^{(\alpha)}(\xi),
$$

so the denominator in the formula given by Theorem 2.1 is, in this case,

$$
\sum_{k=0}^{n-1}\left[L_{k}^{\alpha}(\xi)\right]^{2} k ! /(\alpha+1)_{k}
$$

which may be summed, using [8, p. 188, (9)], to get

$$
-\frac{n !}{(\alpha+1)_{n-1}} L_{n-1}^{\alpha}(\xi)\left[L_{n}^{\alpha}(\xi)\right]^{\prime} .
$$

The numerator is $\mathscr{U}^{T}(\partial H / \partial \tau) \mathscr{U}$, where the $(i, j)$ th element of $\partial H / \partial \tau$ is

$$
-\frac{\sqrt{i+1}}{2 \sqrt{\alpha+i+1}} \delta_{i, j-1}+\delta_{i, j}-\frac{\sqrt{i}}{2 \sqrt{\alpha+i}} \delta_{i, j+1} .
$$

Thus the numerator is equal to

$$
\sum_{k=0}^{n-1} \frac{k !}{(\alpha+1)_{k}} L_{k}^{\alpha}(\xi)\left[L_{k}^{\alpha}(\xi)-L_{k-1}^{\alpha}(\xi)\right]
$$

which, using $[8$, p. 191, (29)], gives

$$
\int_{0}^{\infty} \sum_{k=0}^{n-1} \frac{k !}{(\alpha+1)_{k}} L_{k}^{\alpha}(\xi) L_{k}^{\alpha}(\xi+y) e^{-y} d y .
$$

Using [8, p. 188, (9)], and recalling that $L_{n}^{\alpha}(\xi)=0$, we see that this last expression is equal to

$$
-\frac{n !}{(\alpha+1)_{n-1}} \int_{0}^{\infty} u^{-1} e^{-u} L_{n-1}^{\alpha}(\xi) L_{n}^{\alpha}(\xi+u) d u,
$$


so we finally obtain

$$
\frac{d \xi}{d \alpha}=\frac{1}{\left[L_{n}^{\alpha}(\xi)\right]^{\prime}} \int_{0}^{\infty} u^{-1} e^{-u} L_{n}^{\alpha}(\xi+u) d u .
$$

It is not obvious from this formula that $\xi$ increases with $\alpha, \alpha>-1$. However since we know that this is, in fact, the case we can deduce that

$$
\frac{1}{\left[L_{n}^{\alpha}(\xi)\right]^{\prime}} \int_{0}^{\infty} u^{-1} e^{-u} L_{n}^{\alpha}(\xi+u) d u>0,
$$

where $\xi$ is a zero of $L_{n}^{\alpha}(x), \alpha>-1$. (The numerator obtained above can be shown to be positive for $\alpha \geq 0$ using the Cauchy-Schwarz inequality.)

\section{The Al-Salam-Carlitz polynomials}

Another example illustrating the advantage of our approach is the case of the Al-Salam-Carlitz polynomials $\left\{U_{n}^{(\alpha)}(x)\right\}$; see Chihara [6, p. 196]. These are given by

$$
\begin{gathered}
U_{-1}^{(a)}(x)=0, \quad U_{0}^{(a)}(x)=1, \\
U_{n+1}^{(a)}(x)=\left[x-(1+a) q^{n}\right] U_{n}^{(a)}(x)+a q^{n-1}\left(1-q^{n}\right) U_{n-1}^{(a)}(x),
\end{gathered}
$$

where $a<0,0<q<1$. Although it is not clear whether $U_{n}^{(a)}(x)$ tends to an interesting class of orthogonal polynomials as $q \rightarrow 1^{-}$, it is nevertheless true that the set $\left\{U_{n}^{(a)}(x)\right\}$ has many of the desirable combinatorial properties of a $q$-analogue of the Charlier polynomials [16].

The interval $[a, 1]$ is the true interval of orthogonality of $\left\{U_{n}^{(a)}(x)\right\}$. Hence, by Theorem 2.5 , the tridiagonal symmetric matrix $M$ with

$$
m_{n n}=1-(1+a) q^{n}, \quad m_{n, n+1}=-\sqrt{-a q^{n}\left(1-q^{n+1}\right)}, \quad n=0,1, \ldots,
$$

is positive definite. It will be useful later to replace $a$ by $q^{2} /(4 a)$ giving the positive definiteness of the tridiagonal symmetric matrix $M$ with

$$
\begin{gathered}
m_{n n}=1-\left(1+q^{2} /(4 a)\right) q^{n} \\
m_{n, n+1}=-\sqrt{-q^{n+2}\left(1-q^{n+1}\right) /(4 a)}, \quad n=0,1, \ldots .
\end{gathered}
$$

Now we prove

Theorem 5.1. Let $0<q<1$. The zeros of $\left\{U_{n}^{(a)}(x+a / q)\right\}$ are strictly decreasing functions of $a$ on the interval $-\infty<a<-q^{2}[4(1-q)]^{-1}(<0)$.

Proof. In this case $\beta_{n}=1, \gamma_{n}=(a+1) q^{n}-a / q, \delta_{n}=-a q^{n-1}\left(1-q^{n}\right), \tau=a$, so the elements of the matrix $A$ are given by

$$
a_{n n}=(a+1) q^{n}-a / q, \quad a_{n, n+1}=\sqrt{-a q^{n}\left(1-q^{n+1}\right)}, \quad n=0,1, \ldots,
$$

and the elements of the matrix $-\partial A / \partial a$ are given by

$$
c_{n n}=-q^{n}+1 / q, \quad c_{n, n+1}=\sqrt{q^{n}\left(1-q^{n+1}\right)}(-a)^{-1 / 2} / 2, \quad n=0,1, \ldots
$$


We need to show that, under the given conditions, this matrix is positive definite. We do this by comparing it with (10), using Theorem 2.4. This leads to showing that

$$
\frac{1}{\left(1-q^{n+1}\right)\left(1-q^{n+2}\right)} \leq \frac{1}{\left(1-\left(1+q^{2} /(4 a)\right) q^{n}\right)\left(1-\left(1+q^{2} /(4 a)\right) q^{n+1}\right)} .
$$

But this is true under the given hypothesis $-\infty<a<-q^{2}[4(1-q)]^{-1}$ which is equivalent to $q \leq 1+q^{2} / 4 a$. This completes the proof of Theorem 5.1.

We remark that some restriction on $a$, though probably not as stringent as $-\infty<a<-q^{2}[4(1-q)]^{-1}$, which arose in using our comparison result (Corollary 2.7), is necessary for the positive definiteness of $-\partial A / \partial a$. This can be seen by examining the positivity of the determinant

$$
\left|\begin{array}{cc}
1 / q-1 & \frac{1}{2} \sqrt{(q-1) / a} \\
\frac{1}{2} \sqrt{(q-1) / a} & 1 / q-q
\end{array}\right|
$$

of the first two rows and columns of $-\partial A / \partial a$.

Theorem 5.2. The zeros of $\left\{U_{n}^{(a)}(x \sqrt{-a})\right\}$ increase with $a,-\infty<a<0$.

Proof. In view of Corollary 5.3, to be proved below, we will actually present the formula arising from Theorem 2.1, for the derivatives of the zeros with respect to $a$. It follows from (9) that the orthonormal polynomials

$$
p_{n}(x):=\frac{(-a)^{-n / 2} q^{-n(n-1) / 4}}{\sqrt{(1-q)\left(1-q^{2}\right) \cdots\left(1-q^{n}\right)}} U_{n}^{(a)}(x \sqrt{-a})
$$

satisfy the recursion relation

$$
\begin{aligned}
q^{n / 2} & \left(1-q^{n+1}\right)^{1 / 2} p_{n+1}(x) \\
& =\left(x-(1+a) q^{n} / \sqrt{-a}\right) p_{n}(x)-q^{(n-1) / 2}\left(1-q^{n}\right)^{1 / 2} p_{n-1}(x) .
\end{aligned}
$$

Let $x_{1}<x_{2}<\cdots<x_{n}$ be the zeros of $p_{n}(x)$. For each $j, 1<j<n, x_{j}$ is an eigenvalue of the $n \times n$ truncation of the tridiagonal matrix associated with (11). In this matrix, only the diagonal elements depend on $a$. Furthermore, $\mathscr{U}_{j}=\left(p_{0}\left(x_{j}\right), \ldots, p_{n-1}\left(x_{j}\right)\right)$ is an eigenvector corresponding to the eigenvalue $x_{j}$. Thus Theorem 2.1 gives

$$
\frac{\partial x_{j}}{\partial a}=\sum_{k=0}^{n-1} q^{k} p_{k}^{2}\left(x_{j}\right) \frac{\partial}{\partial a}\left(\frac{1+a}{\sqrt{-a}}\right) / \sum_{k=0}^{n-1} p_{k}^{2}\left(x_{j}\right),
$$

which implies the required result.

Corollary 5.3. Let $x_{1}(a)<x_{2}(a)<\cdots<x_{n}(a)$ be the zeros of $U_{n}^{(a)}(x \sqrt{-a})$. Then

$$
0<x_{j}(b)-x_{j}(a) \leq \frac{1+b}{\sqrt{-b}}-\frac{1+a}{\sqrt{-a}}, \quad a<b<0 .
$$

Proof. In (12), majorize $q^{k}$ by 1 and integrate the resulting differential inequality. 


\section{Meixner polynomials}

The Meixner polynomials

$$
m_{n}(x ; \beta, c)=(\beta)_{n 2} F_{1}\left(\begin{array}{c}
-n,-x \\
\beta
\end{array} \mid 1-c^{-1}\right), \quad \beta>0,0<c<1,
$$

satisfy the three-term recurrence relation

$$
\begin{aligned}
c m_{n+1}(x ; \beta, c)= & {[x(c-1)+n(1+c)+c \beta] m_{n}(x ; \beta, c) } \\
& -n(n+\beta-1) m_{n-1}(x ; \beta, c), \quad n>0,
\end{aligned}
$$

[6, pp. 161 and 176]. One can apply Theorem 2.3 to see that the zeros of $m_{n}(x ; \beta, c)$ increase with $\beta$ when $\beta>1$. The details are similar to our analysis of the associated Laguerre polynomials and will be omitted.

To study the dependence of the zeros of the Meixner polynomials on the parameter $c$, we found it more convenient to use the renormalization

$$
M_{n}(x ; \beta, c):=\frac{(-1)^{n} c^{n / 2}}{\sqrt{n !(\beta)_{n}}} m_{n}\left(\frac{x \sqrt{c}}{1-c} ; \beta, c\right) .
$$

The $M_{n}$ 's are orthonormal polynomials generated by

$$
\begin{aligned}
M_{-1}(x ; \beta, c) & =0, \quad M_{0}(x ; \beta, c)=1 \\
x M_{n}(x ; \beta, c)= & \sqrt{(n+1)(n+\beta)} M_{n+1}(x ; \beta, c) \\
& +[\sqrt{c} \beta+n(1+c) / \sqrt{c}] M_{n}(x ; \beta, c) \\
& +\sqrt{n(n+\beta-1)} M_{n-1}(x ; \beta, c) .
\end{aligned}
$$

As $c \rightarrow 1^{-}$, the zeros of $M_{n}(x ; \beta, c)$ converge to the corresponding zeros of $L_{n}^{(\beta-1)}(x)$, since $[6$, p. 177 or 8, p. $226,(15)]$

$$
\lim _{c \rightarrow 1^{-}} m_{n}\left(\frac{x \sqrt{c}}{1-c} ; \beta, c\right)=n ! L_{n}^{(\beta-1)}(x) \text {. }
$$

We wish to estimate the rate at which the zeros of $M_{n}(x ; \beta, c)$ tend to the corresponding zeros of $L_{n}^{(\beta-1)}(x)$, as $c \rightarrow 1^{-}$.

Let

$$
m_{n, 1}(\beta, c)<\cdots<m_{n, n}(\beta, c) \text { and } l_{n, 1}(\alpha)<\cdots<l_{n, n}(\alpha)
$$

be the zeros of $m_{n}(x ; \beta, c)$ and $L_{n}^{\alpha}(x)$, respectively. We shall denote the zeros of $M_{n}(x ; \beta, c)$ by $M_{n, j}(\beta, c)$, i.e.,

$$
M_{n, j}(\beta, c)=\frac{1-c}{\sqrt{c}} m_{n, j}(\beta, c) .
$$

Theorem 6.1. The quantities $M_{n, j}(\beta, c)$ increase with $c$ on the interval $(n-1) /(\beta+n-1)<c<1$ and converge to $l_{n, j}(\beta-1)$ as $c \rightarrow 1^{-}$.

Proof. The $n \times n$ truncation of the infinite tridiagonal matrix associated with (13) has eigenvalues $M_{n, 1}(\beta, c), \ldots, M_{n, n}(\beta, c)$ and an eigenvector corresponding to $M_{n, j}(\beta, c)$ is

$$
\left\{M_{0}\left(M_{n, j}(\beta, c) ; \beta, c\right), \ldots, M_{n-1}\left(M_{n, j}(\beta, c) ; \beta, c\right)\right\}
$$


We then apply Theorem 2.1 and obtain

$$
\begin{aligned}
\frac{\partial}{\partial c} M_{n, j}(\beta, c)= & \sum_{k=0}^{n-1} \frac{\beta c+k(c-1)}{2 c \sqrt{c}} M_{k}^{2}\left(M_{n, j}(\beta, c) ; \beta, c\right) \\
& \times\left[\sum_{k=0}^{n-1} M_{k}^{2}\left(M_{n, j}(\beta, c) ; \beta, c\right)\right]^{-1} .
\end{aligned}
$$

The coefficients are all positive for the given range of values of $c$. This proves Theorem 6.1.

We now obtain two-sided inequalities for the zeros of the Meixner polynomials.

Theorem 6.2. Let $m_{n, j}(\beta, c)$ and $l_{n, j}(\alpha)$ be as in (14). If $0<c<1$, then

$$
\begin{aligned}
l_{n, j}(\beta-1)-\beta(1-\sqrt{c}) & <\frac{1-c}{\sqrt{c}} m_{n, j}(\beta, c) \\
& <l_{n, j}(\beta-1)-\beta(1-\sqrt{c})+\frac{(n-1)}{\sqrt{c}}(1-\sqrt{c})^{2} .
\end{aligned}
$$

Proof. Observe that (15) holds for all $n>0$ and all $c \in(0,1)$. Since $\beta c \geq$ $\beta c+k(c-1) \geq \beta c+(n-1)(c-1)$, we get

$$
\frac{\beta}{2 \sqrt{c}}>\frac{\partial M_{n, j}(\beta, c)}{\partial c}>\frac{\beta c+(n-1)(c-1)}{2 c \sqrt{c}} .
$$

Integrating this inequality between $c$ and 1 and using $M_{n, j}(\beta, 1)=l_{n, j}(\beta-1)$ we get (16).

\section{Pollaczek polynomials}

The Pollaczek polynomials $P_{n}^{\lambda}(x ; a, b)$ satisfy the recurrence relation [6, $\mathrm{p}$. 184]

$$
\begin{aligned}
(n+1) P_{n+1}^{\lambda}(x ; a, b)= & 2[(n+\lambda+a) x+b] P_{n}^{\lambda}(x ; a, b) \\
& -(n+2 \lambda-1) P_{n-1}^{\lambda}(x ; a, b) .
\end{aligned}
$$

From this we see that the orthonormal Pollaczek polynomials

$$
p_{n}^{\lambda}(x)=p_{n}^{\lambda}(x ; a, b)=\sqrt{\frac{n !(n+\lambda+a)}{(\lambda+a)(2 \lambda)_{n}}} P_{n}^{\lambda}(x ; a, b)
$$

satisfy

$$
\begin{aligned}
x p_{n}^{\lambda}(x ; a, b)= & \frac{1}{2} \sqrt{\frac{(n+1)(n+2 \lambda)}{(\lambda+a+n)_{2}}} p_{n+1}^{\lambda}(x ; a, b) \\
& -\frac{b}{n+\lambda+a} p_{n}^{\lambda}(x ; a, b)+\frac{1}{2} \sqrt{\frac{n(n+2 \lambda-1)}{(\lambda+a+n-1)_{2}}} p_{n-1}^{\lambda}(x ; a, b) .
\end{aligned}
$$


Thus if $\left\{x_{n, i}^{\lambda}(a, b)\right\}$ are the zeros of $p_{n}^{\lambda}(x ; a, b)$, then by Theorem 2.1, (17)

$$
\frac{\partial}{\partial b} x_{n, i}^{\lambda}(a, b)=-\sum_{k=0}^{n-1} \frac{1}{k+\lambda+a}\left[p_{k}^{\lambda}\left(x_{n, i}^{\lambda}(a, b)\right)\right]^{2} / \sum_{k=0}^{n-1}\left[p_{k}^{\lambda}\left(x_{n, i}^{\lambda}(a, b)\right)\right]^{2},
$$

which implies the following theorem.

Theorem 7.1. If $\lambda+a>0$, then the zeros of the Pollaczek polynomials decrease as $b$ increases.

From (17), we obtain, for $\lambda+a>0$,

$$
\frac{1}{\lambda+a+n-1}<-\frac{\partial}{\partial b} x_{n, i}^{\lambda}(a, b)<\frac{1}{\lambda+a} \text {. }
$$

Integrating this between 0 and $b$, we get

$$
\frac{b}{\lambda+a+n-1}<x_{n, i}^{\lambda}(a, 0)-x_{n, i}^{\lambda}(a, b)<\frac{b}{\lambda+a}, \quad \lambda+a>0, b>0 .
$$

The lower bound in (18) seems to be sharp.

We write $x_{n, i}^{\lambda}(a, b)=\cos \left(\theta_{n, i}^{\lambda}(a, b)\right)$, where the zeros of $P_{n}^{\lambda}(x ; a, b)$ satisfy

$$
1>x_{n, 1}^{\lambda}(a, b)>x_{n, 2}^{\lambda}(a, b)>\cdots>x_{n, n}^{\lambda}(a, b)>-1 \text {. }
$$

The Pollaczek polynomials reduce to the ultraspherical ones when $a=b=0$. In this case we have the limiting relation

$$
n \theta_{n, i}^{\lambda}(0,0) \rightarrow j_{\lambda-1 / 2, i}
$$

[21, Theorem 8.1.2]. It is of interest to discover the asymptotic behaviour of the zeros for general $a$ and $b$. R. Askey [1] has conjectured that

$$
n\left[\theta_{n, i}^{\lambda}(a, b)\right]^{2}=2 a+2 b+\frac{1}{4 n} j_{2 \lambda-1, i}^{2}+o(1 / n), \quad n \rightarrow \infty .
$$

Here we are able to get some information in the case $a=0$. In this case, the lower bound in (16) leads to

$$
\frac{b}{\lambda+n-1}+2 \sin ^{2}\left(\frac{1}{2} \theta_{n, i}^{\lambda}(0,0)\right)<2 \sin ^{2}\left(\frac{1}{2} \theta_{n, i}^{\lambda}(0, b)\right) .
$$

Thus we have

$$
\begin{aligned}
n\left[\theta_{n, i}^{\lambda}(0, b)\right]^{2} & >4 n \sin ^{2}\left(\frac{1}{2} \theta_{n, i}^{\lambda}(0, b)\right) \\
& \geq \frac{2 n b}{\lambda+n-1}+4 n \sin ^{2}\left(\frac{1}{2} \theta_{n, i}^{\lambda}(0,0)\right),
\end{aligned}
$$

leading to the asymptotic lower bound

$$
n\left[\theta_{n, i}^{\lambda}(0, b)\right]^{2}>2 b+\frac{2 b(1-\lambda)}{n}+\frac{1}{n} j_{\lambda-1 / 2, i}^{2}+O\left(1 / n^{2}\right),
$$

for sufficiently large $n$ and $\lambda>-1 / 2$. 


\section{REFERENCES}

1. R. Askey, private communication.

2. R. Askey and M. E. H. Ismail, Recurrence relations, continued fractions and orthogonal polynomials, Mem. Amer. Math. Soc. No. 300 (1984).

3. R. Askey and J. Wimp, Associated Laguerre and Hermite polynomials, Proc. Roy. Soc. Edinburgh Sect. A 96 (1984), 15-37.

4. F. V. Atkinson, Discrete and continuous boundary value problems, Academic Press, New York, 1964.

5. T. S. Chihara, Chain sequences and orthogonal polynomials, Trans. Amer. Math. Soc. 104 (1962), 1-16.

6. _ An introduction to orthogonal polynomials, Gordon and Breach, New York, 1978.

7. A. Erdélyi et al., Higher transcendental functions, vol. 1, McGraw-Hill, New York, 1953.

8. _ Higher transcendental functions, vol. 2, McGraw-Hill, New York, 1954.

9. G. Freud, Orthogonal polynomials, English transl., Pergamon Press, New York, 1971.

10. W. Hahn, Über Orthogonalpolynome mit drei Parameteren, Deutsche Math. 5 (1940-41), 373-378.

11. R. Horn and C. Johnson, Matrix analysis, Cambridge Univ. Press, Cambridge, 1985.

12. M. E. H. Ismail, The variation of zeros of certain orthogonal polynomials, Adv. Appl. Math. 8 (1987), 111-118.

13. _ Monotonicity of zeros of orthogonal polynomials, in $q$-Series and Partitions (D. Stanton, ed.), IMA Vol. Math. Appl., vol. 18, Springer-Verlag, New York, 1989, pp. 177-190.

14. M. E. H. Ismail, J. Letessier, D. R. Masson, and G. Valent, Birth and death processes and orthogonal polynomials, Orthogonal Polynomials: Theory and Practice (P. Nevai, ed.), NATO ASI Series, Kluwer Academic Publishers, Dordrecht, Boston, and London, 1990, pp. 229-255.

15. M. E. H. Ismail, J. Letessier, and G. Valent, Linear birth and death models and associated Laguerre polynomials, J. Approx. Theory 56 (1988), 337-348.

16. M. E. H. Ismail, D. Stanton, and D. White, The combinatorics of Charlier polynomials (to appear).

17. M. E. H. Ismail and R. Zhang, On the Hellmann-Feynman theorem and the variation of zeros of certain special functions, Adv. Appl. Math. 9 (1988), 439-446.

18. A. Laforgia and M. E. Muldoon, Some consequences of the Sturm comparison theorem, Amer. Math. Monthly 93 (1986), 89-94.

19. L. Lorch, Elementary comparison techniques for certain classes of Sturm-Liouville equations, Differential Equations (G. Berg, M. Essén, and Å. Pleijel, eds.), Proc. Conf. Uppsala, 1977, Almqvist and Wiksell, Stockholm, 1977, pp. 125-133.

20. A. W. Marshall and I. Olkin, Inequalities: Theory of majorization and its applications, Academic Press, New York, 1979.

21. G. Szegö, Orthogonal polynomials, Amer. Math. Soc. Colloq. Publ., vol. 23, 4th ed., Amer. Math. Soc.,, Providence, R.I., 1975.

22. H. S. Wall, Continued fractions, Van Nostrand, New York, 1948; reprinted, Chelsea, New York, 1967.

23. H. S. Wall and M. Wetzel, Quadratic forms and convergence regions for continued fractions, Duke Math. J. 11 (1944), 373-397.

Department of Mathematics, University of South Florida, Tampa, Florida 33620

Department of Mathematics, York University, North York, Ontario M3J 1P3, Canada 